\title{
Powder X-Ray Diffraction and Electron Microscopy Studies of Polycrystalline $\mathrm{Au}_{2}$ PrIn
}

Robin T. Macaluso, ${ }^{1 *}$ Brian Wells, ${ }^{2}$ Chad Wangeline, ${ }^{2}$ Kenneth Cochran, ${ }^{2}$ Benjamin K. Greve ${ }^{2}$

${ }^{1}$ Department of Chemistry and Biochemistry, University of Texas at Arlington, Arlington, TX 76019

${ }^{2}$ Department of Chemistry and Biochemistry, University of Northern Colorado, Greeley, CO 80639

*Corresponding Author: robin.macaluso@uta.edu

\begin{abstract}
Heusler intermetallic compounds, with a general formula of $X_{2} Y Z$ encompass over 1500 known compounds and are considered strong candidates in thermoelectrics and solar cell applications as well as their more fundamental phenomena such as topological insulators. It is important therefore, to investigate the synthesis and treatment conditions. Polycrystalline samples of $\mathrm{Au}_{2} \mathrm{PrIn}$ were synthesized and characterized by powder X-ray diffraction. Selective etching of impurity phases was followed and studied using secondary and backscatter electron microscopy and powder X-ray diffraction.
\end{abstract}

Keywords: $\mathrm{Au}_{2}$ Prln, Heusler, scanning electron microscopy

Graphical Abstract

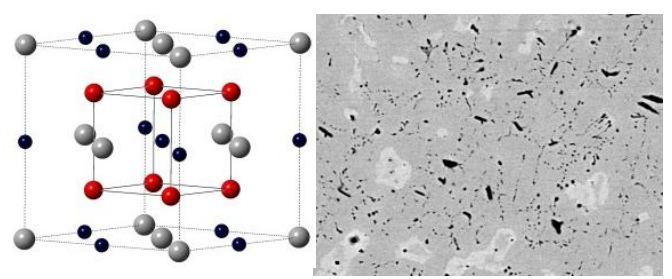

\section{Synopsis}

Polycrystalline samples of the Heusler compound, $\mathrm{Au}_{2} \mathrm{PrIn}$, were synthesized using arc-melting techniques. Powder X-ray diffraction and scanning electron microscopy methods were used to optimize synthesis of polycrystalline $\mathrm{Au}_{2} \operatorname{Pr} \mathrm{Pn}$. Secondary and backscatter electron images showed that nitric acid could be used to remove small amounts of gold-rich impurities. 


\section{Introduction}

Heusler phases are a large class of intermetallic compounds (over 1500) with a 2:1:1 stoichiometry. The crystal structure of Heusler compounds, with a general chemical formula of $\mathrm{X}_{2} \mathrm{YZ}$, is shown in Figure 1 . The $\mathrm{X}, \mathrm{Y}$, and $\mathrm{Z}$ atoms occupy the $8 c, 4 a$ and $4 b$ Wyckoff sites, respectively. The compounds crystallize in the $\mathrm{F} m \overline{3} m$ space group (No. 225) and are described and possess four interpenetrating face-centered cubic sublattices, two of which are occupied by the X metal.

Unlike most intermetallic compounds, Heusler compounds uniquely follow valence electron counts. This has led to an abundance of investigations of Heusler compounds as candidates in thermoelectrics, solar cell, and for their more fundamental phenomena such as topological insulators. Magnetic $X_{2} Y Z$ compounds show various magnetic behavior and properties, such as magneto-optical [1], magnetocaloric [2] and magneto-structural phase transitions [3]. Such phase transitions lead to Heusler compounds as shape-memory alloys. One well-known example is demonstrated in $\mathrm{Ni}_{2} \mathrm{MnGa}$, which undergoes a martensitic transition at 210 and $370 \mathrm{~K}[4,5]$. Shape-memory alloys undergo stress-driven (typically with temperature) martensitic transitions where the low-temperature phase is crystallographically unique from the high-temperature phase and results from a homogeneous lattice deformation. An excellent review of Heusler compounds will provide the reader with further insight into the rich history of Heusler phases [6].

$\mathrm{Ag}_{2} \mathrm{PrIn}$ is a Heusler compound that has been shown to exhibit a rarely observed quadrupolar Kondo effect. The quadrupolar Kondo effect originates from the combination of the $f^{2}$ electron configuration and the cubic symmetry of the $\mathrm{Pr}^{3+}$ ion. Under these conditions, the quadrupolar moments of the $\mathrm{Pr}^{3+}$ hybridize with conduction electrons to produce unusual magnetic and thermodynamic behavior [7-10].

Because the bonding between $\operatorname{Pr}$ and Ag play an important role in its physical behavior, we investigated the synthesis and characterization of $\mathrm{Au}_{2} \operatorname{Pr} \mathrm{In}$. Because $\mathrm{Ag}$ and $\mathrm{Au}$ both belong to Group 11 of the periodic table, we expected that $\mathrm{Au}_{2} \mathrm{PrIn}$ would also form a Heusler phase and that we should be able to make direct comparisons between $\mathrm{Au}_{2} \operatorname{Prln}$ and $\mathrm{Ag}_{2} \mathrm{PrIn}$. In this paper, we report the synthesis of polycrystalline $\mathrm{Au}_{2} \mathrm{PrIn}$ and characterization of these samples by scanning electron microscopy.

\section{Materials and Methods}

\section{1. Synthesis}


$\operatorname{Pr}, \mathrm{Au}$, and In were weighed stoichiometrically and placed on a copper hearth of an arc-melter. The chamber of the arc-melter was then evacuated and back-filled with ultra-pure argon gas three times. With Ar gas flowing through the chamber, the metal pieces were melted into a button and allowed to cool on a water-cooled copper hearth. The button was flipped and melted three times to ensure homogeneity. The resulting button was metallic in luster. No weight loss occurred during the synthesis. A small portion of the button was separated and finely ground in an agate mortar and pestle for powder diffraction experiments. The sample is stable in air.

The pellet was mounted into an epoxy stub. The surface of the pellet was subsequently polished in order of 15, 6, and 3 and 1 micron diamond polishing disks. An optical microscope was used to visually inspect the surface for smoothness before applying the next polishing disk.

A plastic pipette was used to place 1-2 drops of acid onto the polished surface of the $\mathrm{Au}_{2} \mathrm{Prln}$ to etch. Glacial acetic acid and various concentrations of $\mathrm{HNO}_{3}$ were tested. Once the acid was placed onto the surface of the pellet, a timer was started for various durations to determine the optimal time for etching the sample surface. Once the timed etch was complete, the sample surface was immediately rinsed with copious amounts of distilled water and dried in air. The sample was then placed into the vacuum chamber of an SEM for elemental analysis.

\subsection{Powder X-ray Diffraction}

Powder X-ray diffraction (XRD) data were collected on ground samples of $\mathrm{Au}_{2} P$ Prln using a $\mathrm{GBC}$ powder X-ray diffractometer equipped with a Cu Ka $\alpha_{1}$ radiation source $(\lambda=1.54184 \AA$ ). A range of $2 \theta$ values from $10^{\circ}$ to $110^{\circ}$, with a scan rate of $0.1^{\circ} / \mathrm{min}$ and a step size of $0.01^{\circ}$ were used. High resolution XRD data were collected through the mail-in service at the 11-BM beamline at the Advanced Photon Source (APS) at Argonne National Laboratory. Discrete detectors covering an angular range from -6 to $16^{\circ} 2 \theta$ were scanned over a $34^{\circ} 2 \theta$ range, with data points collected every $0.001^{\circ} 2 \theta$ and scan speed of $0.01^{\circ} \%$ s.

Refinements of synchrotron data were carried out using GSAS software package to determine lattice parameters $[11,12]$. All data were collected at room temperature.

\subsection{Scanning Electron Microscopy}

Surfaces of $\mathrm{Au}_{2}$ PrIn were characterized by electron dispersive spectroscopy (EDS) using a JEOL 6610LV Scanning Electron Microscope (SEM), equipped with an Oxford XMax EDS detector with a $20 \mathrm{~mm}^{2}$ window, operated with an accelerating voltage of $20 \mathrm{kV}$ and a working distance of $10 \mathrm{~mm}$. The arc-melted button was cut into four fragments. Several spots on each fragment were analyzed for $45 \mathrm{~s}$, 
Pr.

ImageJ is an open source software program developed by the National Institutes of Health that can evaluate area and pixel statistics of images [13]. ImageJ was applied to SEM images to quantify compositions of the samples.

\section{Results and Discussion}

\subsection{X-ray Diffraction}

LeBail fits were performed on the diffraction data collected at APS (provided in Supplementary Material) to determine the unit cell dimension $a=7.0507(2) \AA$, which is similar to previously reported value of $7.055 \AA$ [14]. It is well-known that diffraction patterns can be used to investigate order or disorder in Heusler compounds. Disorder within the YZ sublattice extinguishes odd superlattice diffraction peaks, e.g., $h+k+I=2 n+1$, while disorder between the $\mathrm{X}$ and YZ sublattice extinguishes $(h+$ $k+I) / 2=2 n+1$, e.g., (200) peaks. Figure 2 shows an X-ray diffraction pattern of the sample as synthesized. The absence of $(h+k+l)=2 n+1$ peaks, e.g., (111) and (331) calculated to be at $2 \theta \sim 5.8^{\circ}$ and $14.7^{\circ}$, respectively, suggests disorder between $\mathrm{Pr}$ and In. Rietveld refinements were not performed because the severe overlap of peaks from the impurity and desired $\mathrm{Au}_{2} \mathrm{Pr} I n$ phases led to inconclusive results. The major portion of the sample can be identified as $\mathrm{Au}_{2} \operatorname{Pr} I n$, but some peaks, i.e., $2 \theta \sim 34^{\circ}$, could not be matched to $\mathrm{Au}_{2} P r I n$ and indicate the presence of an impurity. The impurity peaks can be more easily seen in the Figure 2 inset. Based on the relative peak intensity, it is estimated that this impurity is approximately $10 \%$ of the entire sample volume. We compared the powder patterns of binary compounds to the collected powder pattern, but were unable to successfully identify the composition of the impurity through diffraction.

\subsection{Sample Composition}

In SEM, inelastic scattering of secondary electrons from the incident electron beam varies exponentially with sample depth. Hence, images with high spatial resolution can be obtained. Elastic scattering of backscattered electrons, on the other hand, are responsive to composition contrast. Generally, brighter areas in backscattered images correspond to compositions of higher electron density, and darker areas correspond to compositions of lower electron density. Quantification can be performed with backscattered electron images [15]. Therefore, secondary and backscattered electron images can provide complimentary information about a material. 


\subsection{Etching}

In our first attempt at etching the pellet, glacial acetic acid was applied to the sample surface. After $5 \mathrm{~h}$ and $45 \mathrm{~min}$ of exposure to acetic acid, no changes were observed. In fact, the epoxy mount degraded after some exposure to acetic acid, and the $\mathrm{Au}_{2}$ PrIn sample was remounted into fresh epoxy and re-polished.

A $1 \mathrm{M}$ hydrochloric $(\mathrm{HCl})$ acid solution was then applied to the sample surface. After $2 \mathrm{~h}$, no etching of the sample surface was observed. The sample surface was cleaned with deionized (DI) water and acetone before applying a $1 \mathrm{M}$ nitric acid $\left(\mathrm{HNO}_{3}\right)$ solution.

After $3.5 \mathrm{~h}$ of exposure to $0.10 \mathrm{M}$ and $1 \mathrm{M} \mathrm{HNO}_{3}$ solutions, there were not any observable changes on the sample surface. Applying a $5 \mathrm{M} \mathrm{HNO}_{3}$ solution to the sample, however, resulted in more drastic changes to the surface, which can be seen in Figure 4 . Figures $4 a$ and $4 b$ show the sample surface before and after application of $5 \mathrm{M} \mathrm{HNO}_{3}$ for 3 hours. Backscatter images show valleys in bright regions, while the rest of the surface remains unchanged. EDS spectra were collected to determine that bright 
and intermediate regions correspond to $A u_{3.9(5)} P r_{1.2(3)} \ln _{1.0(3)}$ and $A u_{2.7(5)} \operatorname{Pr}_{1.3(3)} \ln _{1.0(3)}$, respectively. These compositions match the compositions of the sample before etching. Hence application of $\mathrm{HNO}_{3}$ is simply resulting in etching away the Au-rich phase; the sample had not undergone chemical changes.

After an additional hour (for a total of $4 \mathrm{~h}$ ) of exposure to $5 \mathrm{M} \mathrm{HNO}_{3}$, another backscatter image (shown in Figure 4c) was collected. This image was collected from another location on the sample surface (different than the location shown in Figures $4 a$ and $4 b$ ), so we cannot firmly conclude that etching had progressed with time; however, the presence of valleys in the bright regions indicates that etching was not isolated to one small spot on the sample surface. Figure $4 \mathrm{~d}$ shows the same location as Figure $4 \mathrm{c}$ after $5.5 \mathrm{~h}$ of exposure to $5 \mathrm{M} \mathrm{HNO}_{3}$. The valleys in Figure $4 \mathrm{~d}$ are located in the same spots, but are considerably larger than those in Figure 4c. This observation leads us to conclude that etching of the $\mathrm{Au}_{3.9(5)} \operatorname{Pr}_{1.2(3)} \mathrm{In}_{1.0(3)}$ phase was still proceeding.

\section{Conclusion}

Arc-melting synthesis methods yielded two phases detectable by backscattered electron microscopy: $A u_{3.9(5)} \operatorname{Pr}_{1.2(3)} \ln _{1.0(3)}$ and $A u_{2.7(5)} \operatorname{Pr}_{1.3(3)} \ln _{1.0(3)}$. We demonstrated the ability to selectively etch the Au-rich phase, $A u_{3.9(5)} \operatorname{Pr}_{1.2(3)} \ln _{1.0(3)}$, when both $A u_{3.9(5)} \operatorname{Pr}_{1.2(3)} \ln _{1.0(3)}$ and $A u_{2.7(5)} \operatorname{Pr}_{1.3(3)} \ln _{1.0(3)}$ are present in a matrix.

Gold is generally considered to be insoluble in acid. It has been previously shown that small quantitative amounts of gold can be recovered from filtrates of nitric acid that was boiled with solid gold or gold-containing alloys [18]. The ability to dissolve only small amounts of Au allowed us to selectively etch the Au-rich phase of the $A u_{3.9(5)} \operatorname{Pr}_{1.2(3)} \ln _{1.0(3)} / A u_{2.7(5)} \operatorname{Pr}_{1.3(3)} \ln _{1.0(3)}$ mixture. We determined that a minimum concentration of $5 \mathrm{M} \mathrm{HNO}_{3}$ was needed to successfully etch, and that the etching process requires at least $5 \mathrm{~h}$. The etching process was visually followed by secondary and backscatter SEM images. Stronger concentrations of $\mathrm{HNO}_{3}$ and longer exposure times (greater than $5 \mathrm{~h}$ ) could be used to determine optimal etching conditions.

\section{Acknowledgment}

RTM and BDW acknowledge NSF CAREER Award DMR-1541230 for support of this work. Use of the Advanced Photon Source at Argonne National Laboratory was supported by the U. S. Department of Energy, Office of Science, Office of Basic Energy Sciences, under Contract No. DE-AC02-06CH11357.

\section{References}

[1] P.G. van Engen, K.H.J. Buschow, R. Jongebreur, M. Erman, Appl. Phys. Lett., 42 (1983) 202-204.

[2] T. Krenke, E. Duman, M. Acet, E.F. Wassermann, X. Moya, L. Manosa, A. Planes, Nat. Mater., 4 (2005) 450-454. 
[3] R. Kainuma, Y. Imano, W. Ito, Y. Sutou, H. Morito, S. Okamoto, O. Kitakami, K. Oikawa, A. Fujita, T. Kanomata, K. Ishida, Nature, 439 (2006) 957-960.

[4] K. Ullakko, J.K. Huang, C. Kantner, R.C. O’Handley, V.V. Kokorin, Appl. Phys. Lett., 69 (1996) 19661968.

[5] P.J. Webster, K.R.A. Ziebeck, S.L. Town, M.S. Peak, Phil. Mag. B, 49 (1984) 295-310.

[6] T. Graf, C. Felser, S.S.P. Parkin, Prog. Solid State Chem., 39 (2011) 1-50.

[7] A. Yatskar, W.P. Beyermann, R. Movshovich, P.C. Canfield, Phys. Rev. Lett., 77 (1996) 3637-3640.

[8] H. Tanida, S. Takagi, H. S. Suzuki, I. Satoh, T. Komatsubara, J. Phys. Soc. Japan, 75 (2006) 074721.

[9] O. Suzuki, H. Suzuki, H. Kitazawa, G. Kido, Physica B, 359-361 (2005) 926-928.

[10] Y. Sato, Y. Nakamura, H. Morodomi, T. Hasuo, Y. Inagaki, T. Kawae, H.S. Suzuki, M. Mito, T. Kitai, J. Phys.: Conf. Ser., 400 (2012) 032080-032084.

[11] B. Toby, J. Appl. Crystallogr., 34 (2001) 210-213.

[12] A.C. Larson, R.B. Von Dreele, Los Alamos National Laboratory Report LAUR 86-748, Los Alamos National Laboratory, Los Alamos, 2004.

[13] C.A. Schneider, W.S. Rasband, K.W. Eliceiri, Nat. Meth., 9 (2012) 671-675.

[14] M.J. Besnus, J.P. Kappler, M.F. Ravet, A. Meyer, R. Lahiouel, J. Pierre, E. Siaud, G. Nieva, J. Sereni, J. Less Common Met., 120 (1986) 101-112.

[15] J.I. Goldstein, D.E. Newbury, P. Echlin, D.C. Joy, A.D. Romig, Jr., C.E. Lyman, C. Fiori, E. Lifshin, Scanning electron microscopy and X-ray microanalysis, Plenum Press, New York, 1992.

[16] R.E. Honig, RCA Rev., 23 (1962) 567-586.

[17] R.E. Honig, RCA Rev., 30 (1969) 285-305.

[18] F.P. Dewey, J. Am. Chem. Soc., 32 (1910) 318-323. 


\section{Figure Captions}

Figure 1. The crystal structure of $\mathrm{X}_{2} \mathrm{YZ}$ Heusler compounds highlighting two interpenetrating $f c c$ sublattices (marked as dashed lines). Blue, green and red represent $X, Y$, and $Z$ atoms, respectively.

Figure 2. Laboratory powder XRD pattern with most intense peaks of the $\mathrm{Au}_{2} \mathrm{Pr}$ In phase indexed. Peaks of the $\mathrm{Au}_{2} \mathrm{Prln}$ phase, shown as black crosses, match the calculated pattern, shown as red tick marks. Green line represents the background fit. Inset: A zoomed-in portion of XRD pattern. Peaks in the XRD pattern match the calculated pattern, shown as red tick marks. Asterisks mark unidentified impurity phases.

Figure 3. a) Secondary and b) backscatter images taken of the same spot on $\mathrm{Au}_{2} \operatorname{Pr}$ In surface. The dark grey "valleys" in a) are observed as black features in b).

Figure 4. Backscatter images of $\mathrm{Au}_{2} \mathrm{Prln}$ surface after exposure to $5 \mathrm{M} \mathrm{HNO}_{3}$ a) with no exposure to $\mathrm{HNO}_{3}$ and after b) $3 \mathrm{~h}, \mathrm{c}) 4 \mathrm{~h}$, and d) $5.5 \mathrm{~h}$ of exposure to $5 \mathrm{M} \mathrm{HNO}_{3}$. 


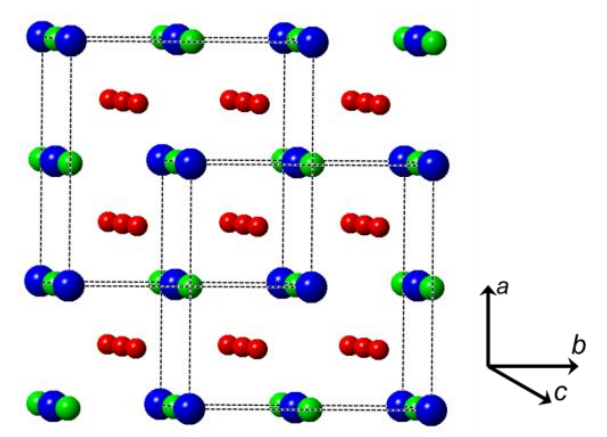

Figure 1. 


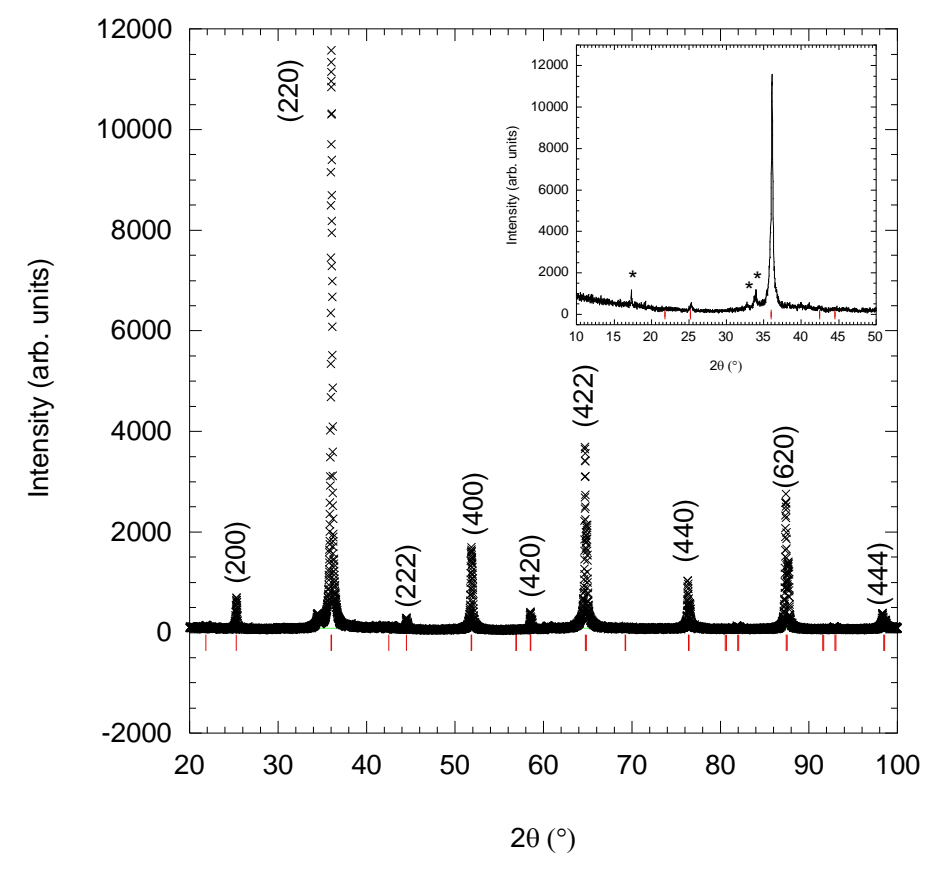

Figure 2.

$2 \theta\left(^{\circ}\right)$

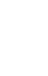

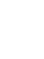

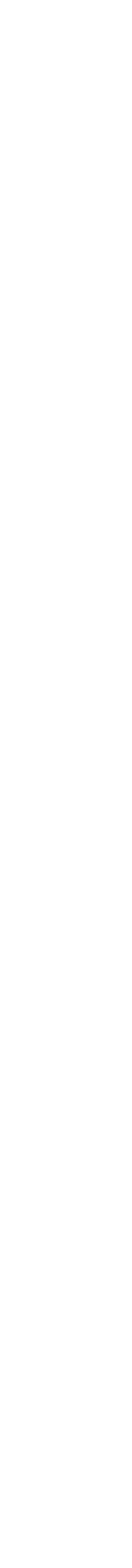

. 


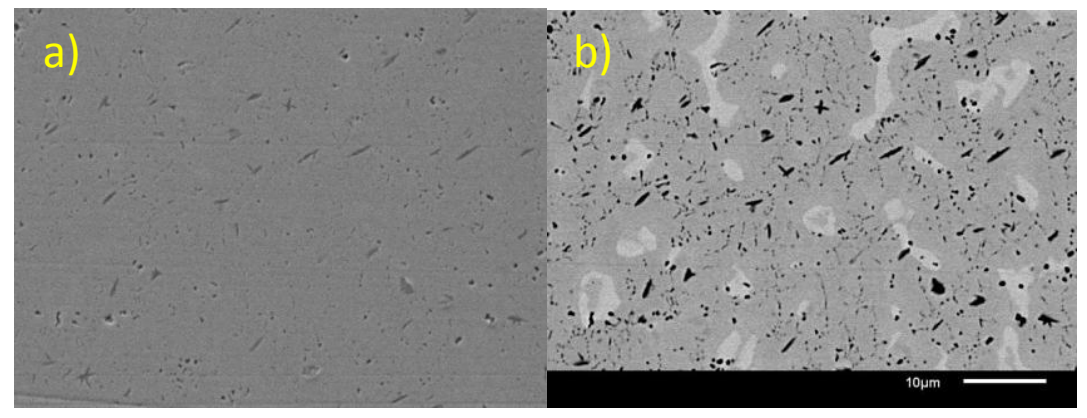

Figure 3. 


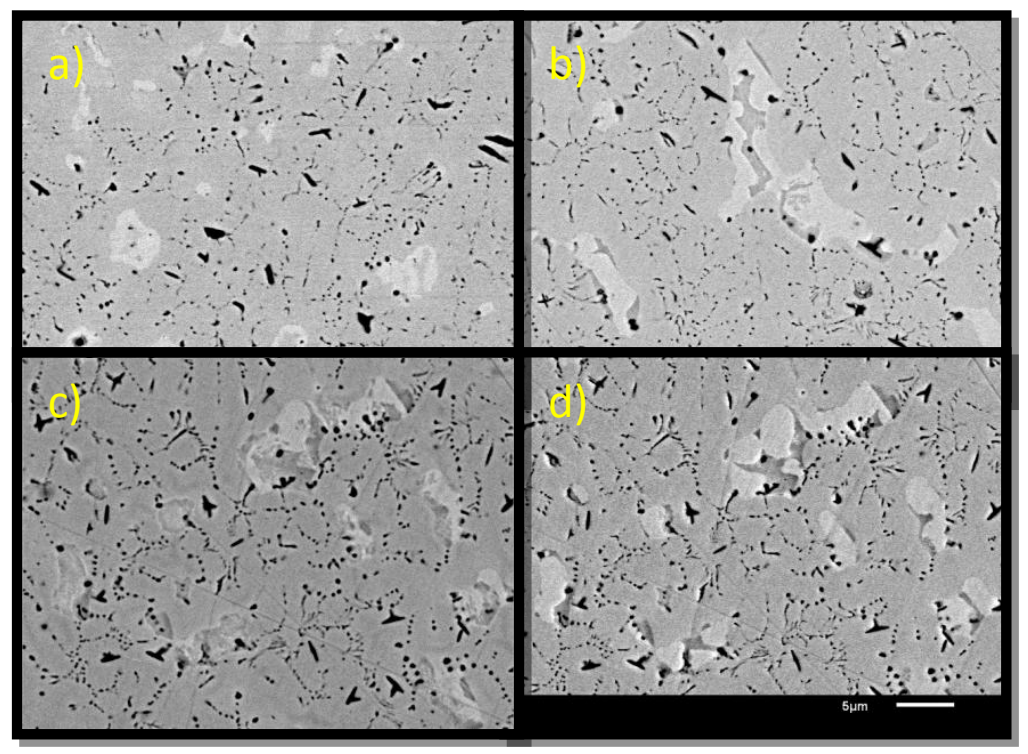

\section{Figure 4.}

